\title{
The effects of perforated cylinders on the vortex shedding on the suppression of a circular cylinder
}

\author{
Engin Pinar ${ }^{1,}$, Tahir Durhasan ${ }^{2}$, Göktürk M. Ozkan ${ }^{2}$, Muhammed M. Aksoy ${ }^{2}$, Huseyin Akilli ${ }^{2}$, Besir Sahin ${ }^{2}$ \\ ${ }^{1}$ Department of Mechanical Engineering, Faculty of Ceyhan Engineering, Cukurova University, 01950, Adana/ Turkey \\ ${ }^{2}$ Department of Mechanical Engineering, Faculty of Engineering and Architecture, Cukurova University, 01330, Adana/ Turkey
}

\begin{abstract}
The aim of this study is the control of unsteady vortical flow occurred downstream of a circular cylinder located in shallow water flow using concentrically located outer perforated cylinder. The porosities, $\beta$ have been changed between 0.1 and 0.8 in the present study. The increments of porosity $\beta$ were taken as 0.05 in the range of 0.1 and 0.8 with a hole diameter of $\mathrm{d}=10 \mathrm{~mm}$. The ratio of inner cylinder diameter to outer cylinder diameter, $\mathrm{D}_{\mathrm{i}} / \mathrm{D}_{\mathrm{o}}$ was selected as $0.25,0.3,0.4,0.5$ and 0.6 the inner cylinder diameter is $D_{i}=50 \mathrm{~mm}$ where the outer cylinder diameter is $D_{d}=100 \mathrm{~mm}$. Experiments were performed at a constant depth of the water level as $\mathrm{h}=50 \mathrm{~mm}$ (half of the outer cylinder diameter). Free stream velocity was taken as $U_{\infty}=100 \mathrm{~mm} / \mathrm{s}$ corresponding to a Reynolds number of Re $D_{0}=10000$ based on the outer cylinder diameter. It has been observed that the inner circular cylinder was highly affected by the existence of surrounding outer perforated cylinders. It is observed that the intensity of Reynolds shear stress correlating, $<\mathrm{u}^{\prime} \mathrm{v}^{\prime} / \mathrm{U}^{2}>$ is completely attenuated in the region both downstream of concentric cylinder and between the concentric cylinders. It is determined from the experiments that porosity, $\beta=0.55$ is the most effective parameter for control of flow structure that is occurred from the inner cylinder.
\end{abstract}

\section{Introduction}

A bluff body such as a cylinder which is placed in a flow alters the general characteristics of the flow structure.. This alteration causes more complex and unsteady flow characteristics like resonance and highamplitude of fluctuating forces and have important implications for flow-induced vibration and noise generation. The source of vibrations and fluctuating forces is the flow separation followed by vortex shedding after a critical value of Reynolds number. The situation is of practical interest in many engineering applications as chimney stacks, long- span bridges, marine structures, tall buildings and towers etc. and has been steadily investigated by the researchers Bearman, $[1,2,3,4,5]$; Matsumato [6]; Williamson [7]; Williamson and Govardhan, $[8,9]$. In order to reduce or eliminate the adverse effects of vortex-induced vibrations (VIV), it is inevitably important to control the flow structure around the bluff body and/or alter the mechanical properties of the material used in terms of damping and natural frequency. Since the material enhancement might be high-priced, various techniques about the flow control have been studied extensively in recent years. Every et al. [10] stated in their review that the flow can be controlled by means of an alteration of the structural profile in order to affect the separation of the boundary layer to prevent the occurrence of vortex shedding along the length of the structure or alter the formation of the wake vortices and their interactions.
Unsteady flow formed downstream of cylinder causes serious problems in many engineering applications. In order to solve the problems mentioned, vortices should be suppressed using various flow control techniques. Price [11] investigated various porous shrouds (a higher diameter cylinder placed coincidently around an inner bare cylinder to understand their effects on suppression of the vortex-excited vibrations. He concluded that the vibration suppression can be achieved by shrouded cylinders. Perforated cylinder shrouds on the flow control has been started to be investigated by other researchers. Every et al. [10] performed a review to point out the practical aspects of vortex excited structural vibrations in a marine environment with particular emphasis on methods of their suppression. Molin [12] worked on a numerical study about perforated shrouds used to reduce vortex- induced vibrations of cylinders. The drag reduction of the circular cylinder was investigated experimentally by Wang et al. [13] in terms of the pressure measurement at a Reynolds number of 82,000 based on the cylinder diameter. A rod was placed upstream of the cylinder to control the flow around the cylinder. The interaction of the rod with the flow around the cylinder was examined to realize the drag reduction mechanism with an upstream rod. Barranow et al. [14] performed a numerical study for controlling of wake flow around a circular cylinder encased in a thin cylindrical perforated sheath. Their studies were carried out unsteady two-dimensional laminar flow conditions. They demonstrated that diverting a part of the flow from

Corresponding author: epinar@,cu.edu.tr 
the front stagnation region to the perforation orifices, generating there a flow stabilising the flow field. Sahin [15] carried out an experimental study using perforated plate as a flow control device. He concluded that nonuniformity in the velocity is increased as the porosity is decreased. Turhal and Cuhadaroglu [16] performed experiments to investigate some aerodynamic parameters of the flow around perforated-surface square, horizontal, and diagonal cylinders. The surface pressures of the perforated surface square cylinders were measured for the cases of injections through the top, rear, top-rear, and all surfaces separately at Re values of 10,000, 16,000, and 24,000. The $C_{D}$ and $S t$ were determined based on pressure coefficient measurements and vortex shedding frequency measurements, respectively. Their experimental results showed that the injection through the various surfaces of a square cylinder has some effects on the aerodynamic characteristics of the square cylinder, depending on the position of the perforated surface. They concluded that $C_{D}$ of a horizontal square cylinder can be reduced by injection through all the surfaces, and for a diagonal square cylinder; it also can be reduced by injection through the rear surfaces. They also revealed that the vortex shedding frequency of the flow around a horizontal square cylinder can also be decreased by high-momentum injection through the rear surface. Gozmen et.al. [17] conducted experimental work on the control of vortical flow of circular cylinder using a splitter plate having different lengths and heights: how a submerged attachment changes the flow structure downstream of bluff bodies in shallow water flow and the possibility of contamination dispersion in transverse direction in shallow flows, due to Karman vortices, be prevented by suppressing the flow oscillations downstream of a bluff body. They found that as the ratio of $h_{p} / h_{w}$ increases, the effect of the splitter plate on the suppression of the vortex shedding increases. Flow characteristics and examination of spectra indicate that Karman vortex shedding is attenuated pronouncedly for the cases of $\mathrm{L} / \mathrm{D} \geq 1$ and $\mathrm{hp} / \mathrm{hw} \geq 0.75$. The transverse Reynolds normal stress is more effective on the attenuation of turbulent kinetic energy than the stream wise Reynolds normal stress, $<u^{\prime} u^{\prime} / U^{2}>$. The value of peak transverse Reynolds normal stress, $<\mathrm{u}^{\prime} \mathrm{u}^{\prime} / \mathrm{U}^{2}>$ is reduced to $90 \%$ of that of the bare cylinder at most. Oruc et. al. [18] were investigated suppression of asymmetric flow behavior downstream of two side-by-side circular cylinders with a splitter plate in shallow water. The results revealed that splitter plate had little effect on the flow structure for $\mathrm{L} / \mathrm{D}<3$ such that the wake structure downstream of each of the cylinders was narrow and wide. However, the symmetrical flow pattern was noticed downstream of the cylinders for $\mathrm{L} / \mathrm{D}$ $=3$. In these cases, the gap-flow remained unbiased and it was parallel to the flow axis. The peak concentration of Reynolds shear stress, $\left\langle\mathrm{u}^{\prime} \mathrm{v}^{\prime} / \mathrm{U}^{2}\right\rangle$ also diminished substantially. Increasing splitter plate length also caused the location of peak magnitude of $\left\langle u^{\prime} v^{\prime}\right\rangle$ to move slightly in downstream direction as well as elongation of vorticity contours in stream wise direction. The deflection of the wake and thereby the instability of the wake was considerably prevented with the presence of the splitter plate for which resulted in two well symmetric, stable wakes having approximately the same order of magnitudes of vortex shedding frequencies around the cylinders. Pinar et al. [19] stated in their review that the experimental investigations were carried out in order to have detailed information on the flow structure around perforated cylinders using high-image density Particle Image Velocimetry technique in shallow water flow. In order to analyze the effect of porosity, $\beta$ on the flow structure, the porosities in the range of 0.1 $\leq \beta \leq 0.8$ with an increment of 0.1 were used and the results were compared with the bare cylinder case by means of velocity and vorticity contours, turbulent kinetic energy, Reynolds shear stress and streamline topologies. It was concluded that the porosity, $\beta$ had a substantial effect on the control of large-scale vortical structures downstream of the cylinder in which the shear layers were elongated, fluctuations were significantly attenuated and formation of Karman Vortex Street was successfully prevented by the use of perforated cylinders.

\section{Material and method}

All experiments were carried out in the test section of water channel which has $8000 \mathrm{~mm}$ length $\mathrm{x} 1000 \mathrm{~mm}$ wide $x 750 \mathrm{~mm}$ deep was constructed from a $15 \mathrm{~mm}$ thick transparent plexiglass sheet with upstream and downstream fiberglass reservoirs. Experiments have been studied in a uniform flow by means of both flow visualization with laser illumination of Rhodamine dye technique and digital particle image velocimetry technique (PIV). Visualization of the experimental results were recorded with a high speed SONY 9.2 mega pixels HDR-PJ670 handycam type digital video recorder to examine the flow behaviors in detail. The video camera is used to capture the instantaneous video images of the vortex flow structures. Each experiment is recorded approximately three minutes. The images were analyzed with frame grabber. Digitized images were enhanced for analysis using Adobe Photoshop software. Particle Image Velocimetry technique was used to measure the velocity vector fields. The PIV technique is one of the most reliable methods for flow velocity measurement in the modern fluid mechanics and aerodynamics. By this measurement technique, twodimensional instantaneous velocity profiles downstream of the cylinder were measured and flow properties were defined by quantitative images such as average vorticity, streamline topologies, and turbulence statistics of the flow. Flow characteristics were examined by the PIV technique in the annular region between the inner and outer cylinders at different values of porosity $(\beta=0.25$, $0.4,0.55,0.65$ and 0.8$)$ and diameter ratios $\left(\mathrm{D}_{\mathrm{i}} / \mathrm{D}_{\mathrm{o}}=0.25\right.$, $0.3,0.4,0.5$ and 0.6). Figure 1 shows schematic representation for inner cylinder, perforated outer cylinder. 


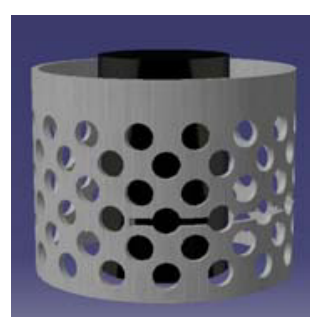

Fig 1. Schematic representation for inner cylinder, perforated outer cylinder.

The porosity ratio, $\beta$, is defined as the ratio of the gap area on the body to the whole body surface area. Perforated outer cylinder is manufactured from metal plate of $1 \mathrm{~mm}$ thickness. For $2 \mathrm{~mm}$ thick laser sheets' illumination of the region between perforated outer cylinder and inner cylinder, 180 degree $1 \mathrm{~mm}$ thick groove is slotted behind the perforated outer cylinder according to flow direction. Diameter ratio, $D_{i} / D_{o}$, is the ratio of inner cylinder diameter to the outer cylinder diameter.

Figure 2 shows representation of water channel. The total depth of the water in the channel was adjusted to a $600 \mathrm{~mm}$ height. All experiments were carried out above a platform, having a length of $2300 \mathrm{~mm}$ to obtain fully developed shallow flow conditions. The water height between the base of the platform and the free surface was adjusted as $50 \mathrm{~mm}$ which corresponds to half of the outer cylinder diameter $\left(D_{0}=100 \mathrm{~mm}\right)$. Free surface velocity is $U=100 \mathrm{~mm} / \mathrm{s}$, which corresponds to the Reynolds number of $\operatorname{Re} D_{0}=10000$ based on the outer cylinder diameter. The measurements were performed and the data were processed using Dantec Dynamics PIV system and Flow Manager Software installed on a computer. The measurement field was illuminated by a thin and an intense laser light sheet by using a pair of double-pulsed Nd: YAG laser units each having a maximum energy output of $120 \mathrm{~mJ}$ at $532 \mathrm{~nm}$ wavelength. The laser sheet was oriented parallel to the bottom surface of the water channel and the experiments were carried out at the midsection of the water height. The image capturing was performed by an 8-bit crosscorrelation CCD camera having a resolution of $1600 \mathrm{x}$ 1200 pixels, equipped with a Nikon AF Micro 60 f/2.8D lens. In the image processing, 32x32 rectangular interrogation pixels were used and an overlap of $50 \%$ was employed. A total of $7128(99 \times 72)$ velocity vectors were obtained for an instantaneous velocity field at a rate of 15 frames per second. The time interval between pulses was $1.5 \mathrm{~ms}$ for all experiments and the thickness of the laser sheet illuminating the measurement plane was approximately $2 \mathrm{~mm}$. The time interval and the laser sheet thickness were selected such that the maximum amount of particle displacement in the interrogation window was obtained.

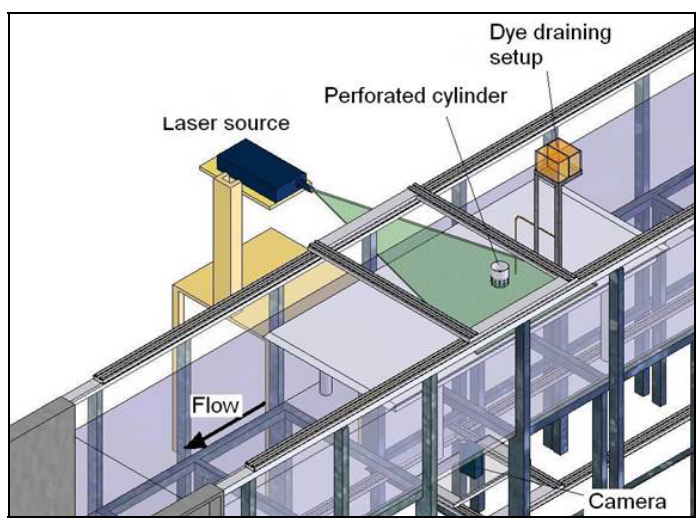

Fig 2. Schematic representation of water channel

\section{Result and Discussion}

First of all, flow characteristics for the bare cylinders are investigated in order to have a reference for the controlled cases. Instantaneous dye images for the bare cylinders case obtained using the flow visualization technique for the inner cylinders are shown in Figure 3. The separated and recirculating flow in the near wake region with the help of visualizing is clearly seen in the case of bare cylinders. It is seen from the dye picture that alternating vortex shedding and the well-known Karman Vortex Street is evident downstream of the cylinders. It is shown that the Von Karman vortex street formed along the downstream of the cylinders have a certain frequency which decreases by increasing diameters of the bare cylinders. The wake is highly unsteady and large eddies or vortices are shed from the downstream region of the cylinders. The large eddies are formed at a regular frequency cause pressure disturbances in the flow. In order to control the near wake region of inner cylinder (bare cylinder), perforated cylinders having various porosity, $\beta$ were used as a control parameters.

Figure 4 presents the result of dye visualization experiments for the porosity, $\beta=0.55$. of the perforated cylinder. Von Karman vortices are controlled easily for low diameter ratios $\left(\mathrm{D}_{\mathrm{i}} / \mathrm{D}_{\mathrm{o}}\right)$ due to the existence of outer perforated cylinder. Kelvin-Helmholtz vortices appear along the shear layer of the concentric cylinders. It can be seen clearly from dye experiments that diameter ratio is an important parameter on the control of Karman vortices formed by the inner cylinder or outer perforated cylinder. It is determined from the dye experiments that porosity, $\beta$ and diameter ratio of inner and outer cylinders, $D_{i} / D_{0}$ are the two significant parameters on the control of flow structure generated by the inner cylinder. The effect of these parameters on the flow structure formed downstream of the inner cylinder is determined in detail with the Particle Image Velocimetry Technique (PIV).

Figure $5 \mathrm{a} 5 \mathrm{~b}$ show the variation of maximum Reynolds shear stress, $<\mathrm{u}^{\prime} \mathrm{v}^{\prime} / \mathrm{U}^{2}>$ values that occurs downstream of the different bare cylinders diameter and concentric cylinder with different diameter ratios, respectively. As it can be seen from figure 5b, maximum values of Reynolds shear stress, $<u^{\prime} v^{\prime} / U^{2}>$ decrease 


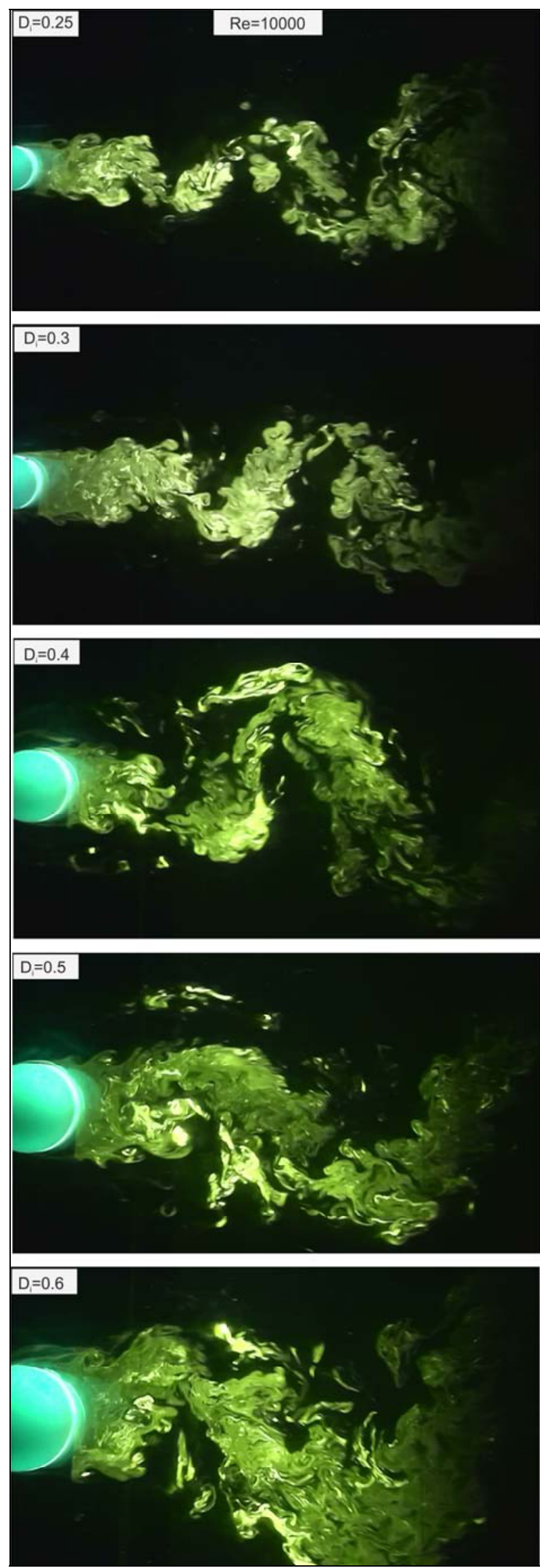

Fig 3. Dye visualization of flow structure around inner (bare) cylinders at $\mathrm{Re}=10000$
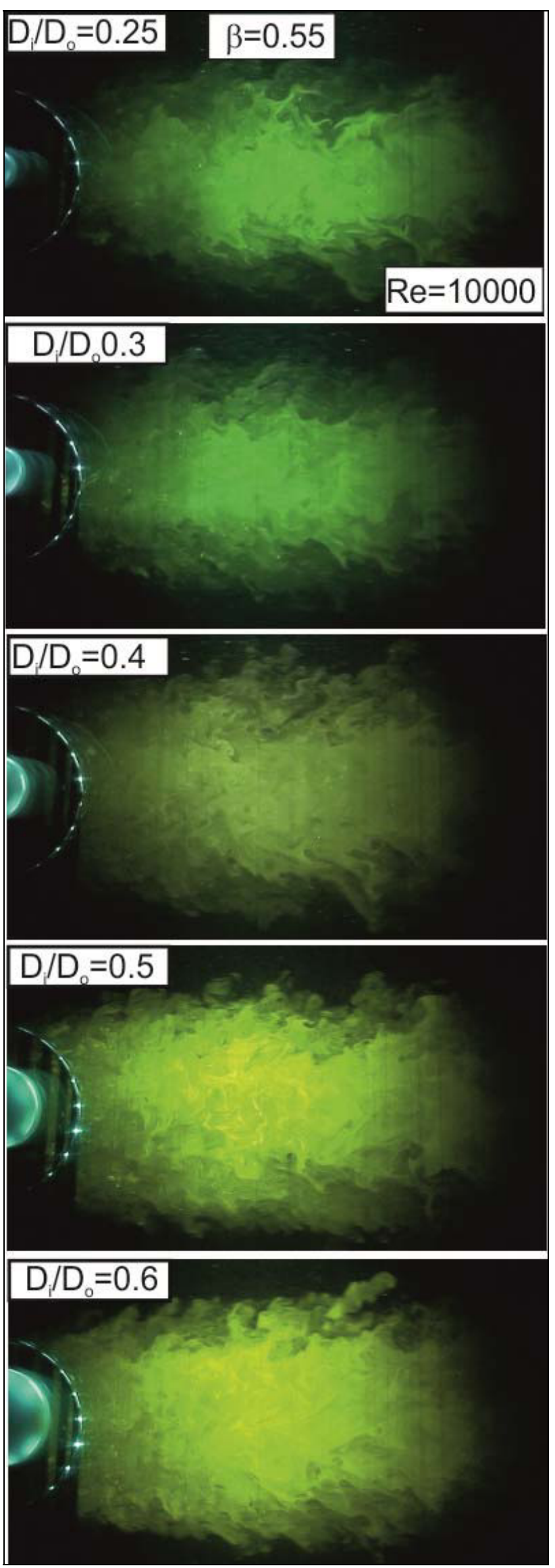

Fig 4. Dye visualization of flow structure downstream of concentric cylinders for various diameter ratios at $\mathrm{Re}=10000$ and porosity, $\beta=0.55$ 
with increasing porosity, $\beta$ up to porosity, $\beta=0.55$ for all diameter ratio. There are sudden increases in the maximum values of Reynolds shear stress, $<\mathrm{u}^{\prime} \mathrm{v}^{\prime} / \mathrm{U}^{2}>$ for $\beta \geq 0.6$ cases for the diameter ratios of 0.5 and 0.6 . Experiments reveal that outer perforated cylinders are significantly effective to reduce values of maximum Reynolds shear stress, $\left\langle\mathrm{u}^{\prime} \mathrm{v}^{\prime} / \mathrm{U}^{2}\right\rangle$ compared to the bare cylinders case in Figure $5 \mathrm{a}$ and $5 \mathrm{~b}$. Formations of the Karman vortices are suppressed and so that a vortical flow structure downstream of the cylinder with a certain frequency does not exist anymore. The situation indicates that the flow structure changes dramatically.

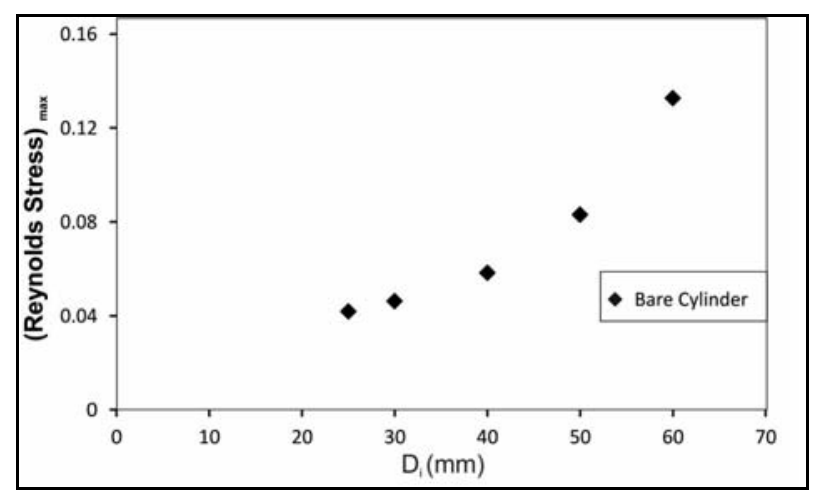

Fig 5a. Variation of Reynolds Shear stress $\max _{\max },\left\langle\mathrm{u}^{\prime} \mathrm{v}^{\prime} / \mathrm{U}^{2}>\right.$ with the downstream of bare cylinder diameter.

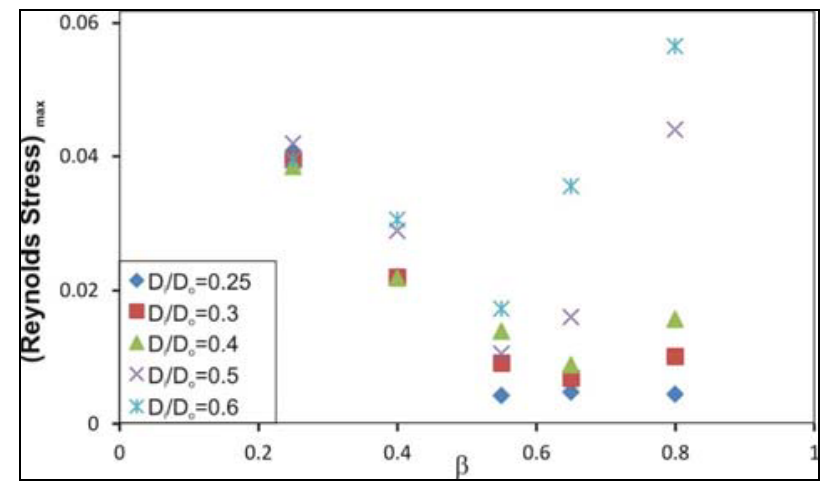

Fig 5b. Variation of Reynolds Shear stress $s_{\max },<\mathrm{u}^{\prime} \mathrm{v}^{\prime} / \mathrm{U}^{2}>$ with porosity, $\beta$, downstream of concentric cylinder.

After this part of the study, all attention was focused on the flow structures between inner and outer cylinder. Especially in smaller diameter ratios, the flow structure in the region between the inner and outer cylinders was investigated in terms of time-averaged Reynolds shear stress, $<\mathrm{u}^{\prime} \mathrm{v}^{\prime} / \mathrm{U}^{2}>$ and streamlines, $\langle\Psi\rangle$, superposed with the vorticity contours $\langle\omega\rangle$ in order to understand whether unsteady Karman vortices are formed or not. The time-averaged streamline topologies, $<\Psi>$, superposed with the vorticity contours between inner and outer perforated cylinders for various porosities, $\beta$ at $D_{i} / D_{0}=0.25$ are given together in Figure 6 The minimum and incremental values of time-averaged vorticity contours, $\langle\omega\rangle$ were taken as \pm 2 and 2 . For low porosity, $\beta$ values, it is seen that the separated flow region in the downstream of the inner cylinder expands in lateral direction which is perpendicular to the main flow direction. Width of the separated flow region decreases with the increasing porosity $\beta$. It is clearly seen from the patterns of streamlines, $\langle\Psi>$ that two foci $\left(F_{1}\right.$ and $\left.F_{2}\right)$ are formed in the downstream of inner cylinder. These flow structures are also observed for the bare cylinder case. Locations of the critical points (foci, F) move toward the outer perforated cylinder from the inner cylinder with increasing porosity, $\beta$. Figure 7 shows the vorticity, $\langle\omega\rangle$ contours and streamlines, $\langle\Psi\rangle$ obtained for $\mathrm{D}_{\mathrm{i}} / \mathrm{D}_{\mathrm{o}}=0.3$. The results obtained from the previous diameter ratio, $\mathrm{D}_{\mathrm{i}} / \mathrm{D}_{\mathrm{o}}=0.25$ are also valid for this case. However, the results obtained for $D_{i} / D_{0}=0.6$ differ from the result of low diameter ratios. The timeaveraged streamline topologies, $\langle\Psi\rangle$, superposed with the vorticity contours between inner and outer perforated cylinders are shown in Figure 8 for $\mathrm{D}_{\mathrm{i}} / \mathrm{D}_{\mathrm{o}}=0.6$ case. When the vorticity, $\langle\omega\rangle$ contours are investigated, the peak magnitude of vorticity, $\langle\omega\rangle$ formed due to inner cylinder increases with increasing the porosity, $\beta$. When the streamlines, $\langle\Psi\rangle$, are investigated, it is seen that critical points named as focus, $F$ does not occur in between the inner and outer perforated cylinders due to the small gap ratio. Flow structure between inner and outer perforated cylinders has changed significantly.

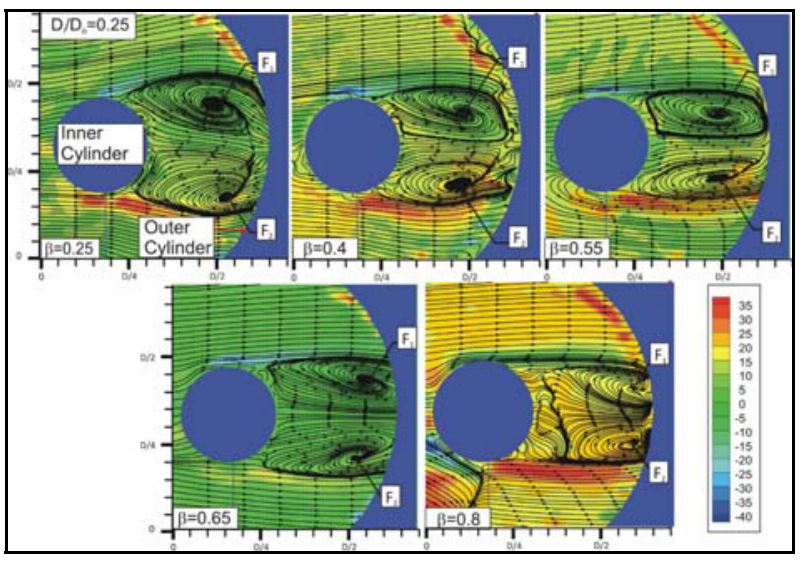

Fig 6. The time-averaged streamline topologies, $\langle\Psi>$ superposed with the vorticity contours $\langle\Delta \omega\rangle=2$ between inner and outer perforated cylinders for various porosities, $\beta$ at $\mathrm{D}_{\mathrm{i}} / \mathrm{D}_{\mathrm{o}}=0.25$

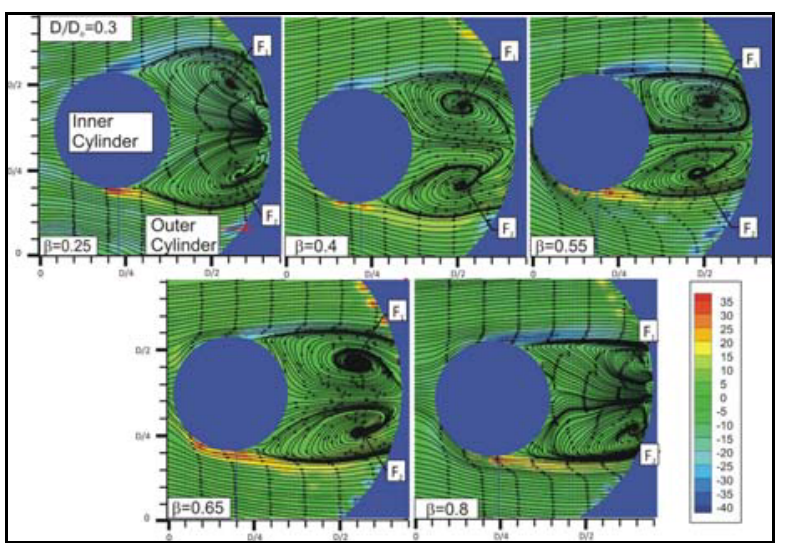

Fig 7. The time-averaged streamline topologies, $\langle\Psi\rangle$ superposed with the vorticity contours $\langle\Delta \omega\rangle=2$ between inner and outer perforated cylinders for various porosities, $\beta$ at $\mathrm{D}_{\mathrm{i}} / \mathrm{D}_{\mathrm{o}}=0.3$ 


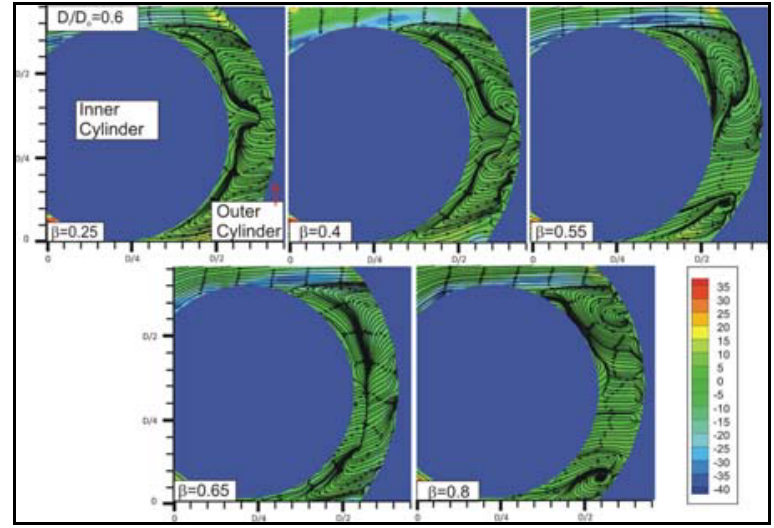

Fig 8. The time-averaged streamline topologies, $\langle\Psi\rangle$ superposed with the vorticity contours $\langle\Delta \omega\rangle=2$ between inner and outer perforated cylinders for various porosities, $\beta$ at $\mathrm{D}_{\mathrm{i}} / \mathrm{D}_{\mathrm{o}}=0.6$.

The variation of the maximum values of Reynolds shear stresses, $<u^{\prime} v^{\prime} / U^{2}>$ in the region between inner and outer cylinders with the porosity, $\beta$ is shown in Figure 9. There is a significant increase in maximum value of Reynolds shear stress, $<\mathrm{u}^{\prime} \mathrm{v}^{\prime} / \mathrm{U}^{2}>$ for $\beta=0.8$. The maximum values of Reynolds shear stress, $<\mathrm{u}^{\prime} \mathrm{v}^{\prime} / \mathrm{U}^{2}>$ were found in the other geometric conditions to be close to each other and independent of the diameter ratio, $\mathrm{D}_{\mathrm{i}} / \mathrm{D}_{\mathrm{o}}$.

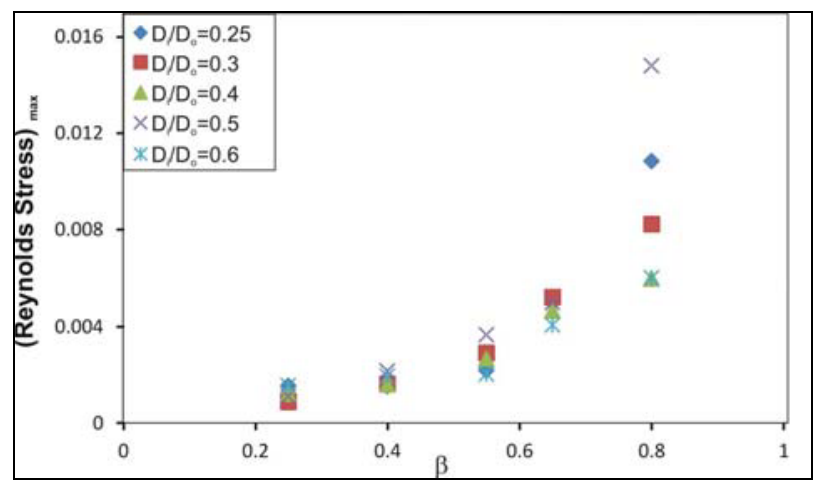

Fig 9. Variation of Reynolds Shear stressmax, $\left\langle\mathrm{u}^{\prime} \mathrm{v}^{\prime} / \mathrm{U}^{2}\right\rangle$ with the porosity, $\beta$, at the region between inner bare cylinder and outer perforated cylinder.

When Figures 5a, 5b and 9 are compared with each other it can be seen that the existence of the outer cylinder is determined to be highly effective on the reduction of the maximum value of Reynolds shear stress, $<\mathrm{u}^{\prime} \mathrm{v}^{\prime} / \mathrm{U}^{2}>$ intensities in the region between the concentric cylinders. In the case of bare cylinder, the increase of cylinder diameter magnitudes the maximum values of Reynolds shear stress, $<\mathrm{u}^{\prime} \mathrm{v}^{\prime} / \mathrm{U}^{2}>$ significantly, but maximum Reynolds shear stress, $<\mathrm{u}^{\prime} \mathrm{v}^{\prime} / \mathrm{U}^{2}>$ formed in the region between the inner and outer perforated cylinder is not affected by the diameter of inner cylinder (except for $\beta=0.8$ ). Due to excessive number of the hole on the perforated cylinder on the cause of this condition is the loss of effect on the flow structure of the perforated cylinder. For small diameter ratios $\mathrm{D}_{\mathrm{i}} / \mathrm{D}_{\mathrm{o}}=$
0.25 and 0.3 and low porosity, $\beta=0.25$, the $\max$ Reynolds shear stress, $\left\langle\mathrm{u}^{\prime} \mathrm{v}^{\prime} / \mathrm{U}^{2}\right\rangle$ value in the downstream of the concentric cylinders has substantially the same value obtained from inner cylinders.

\section{Conclusion}

The effects of porosity, $\beta$ and diameter ratio, $D_{i} / D_{o}$ were investigated and results were discussed to find out the optimum cases for effective flow control. When the perforated cylinder takes place around the bare cylinder, experiments reveal that the flow structure in the region both downstream of concentric cylinder and between the concentric cylinders is significantly affected compared to the bare cylinder case. We understood from qualitative and quantitative experiments that diameter ratios $\left(D_{i} / D_{0}\right)$ does not have a significant influence on the flow structure for region between the inner and outer perforated cylinder unlike downstream of concentric cylinder.

In conclusion, maximum values of Reynolds shear stress, $<\mathrm{u}^{\prime} \mathrm{v}^{\prime} / \mathrm{U}^{2}>$ formed between the inner and outer cylinders have a lower value than the one occurs downstream of bare cylinder or outside of the concentric cylinder which indicates that the volume flow rate entering fluid through the perforated cylinder becomes lower due to pressure increase annular region.

It is determined from the experiments that porosity, $\beta=0.55$ suggestible parameter for reduction of magnitude of Reynolds Shear Stress correlations, $\left\langle\mathrm{u}^{\prime} \mathrm{v}^{\prime} / \mathrm{U}^{2}\right\rangle$ and control of flow structure generated by the inner cylinder both in the region of downstream of concentric cylinder and between inner and outer cylinders.

\section{Acknowledgments}

The authors would like to acknowledge the financial support of this work by the Scientific and Technology Research Council of Turkey under contract no 109R001.

\section{References}

1. G.R.S. Assi, P.W. Bearman, N. Kitney, Journal of Fluids and Structures, Low drag solutions for suppressing vortex-induced vibration of circular cylinders, 25, 666-675 (2009).

2. G.R.S. Assi, P.W. Bearman, N. Kitney, M.A. Tognarelli, Journal of Fluids and Structures, Suppression of wake-induced vibration of tandem cylinders with free-to-rotate control plates, $\mathbf{2 6}$, 1045-1057 (2010).

3. P.W. Bearman, Journal of Fluids and Structures, Circular cylinder wakes and vortex-induced vibrations. 27, 648-658 (2011).

4. P.W. Bearman, Journal of Fluid Mechanics, Understanding and predicting vortex-induced vibrations. 634, 1-4 (2009).

5. P.W. Bearman, Annual Review of Fluid Mechanics Vortex shedding from oscillating bluff bodies. 16, 195-222(1984). 
6. M. Matsumato, Journal of Fluids and Structures, Vortex shedding of bluff bodies, 13,791-811 (1999).

7. C.H.K. Williamson, Annual Review of Fluid Mechanics, Vortex dynamics in the cylinder wake. 28, 477-539 (1996).

8. C.H.K. Williamson, R. Govardhan. Annual Review of Fluid Mechanics, Vortex-induced vibrations, 36, 413-455 (2004).

9. C.H.K. Williamson, R. Govardhan, Journal of Wind Engineering and Industrial Aerodynamics, $A$ brief review of recent results in vortex-induced vibrations. 96, 713-735 (2008).

10. M.J. Every, R. King, D.S. Weaver., Ocean Engineering. Vortex-excited vibrations of cylinders and cables and their suppression, 9, 135-157 (1982).

11. P. Price, J. Eng. Mech. Div., Am. Soc. Civ. Eng., Suppression of the fluid-induced vibration of circular cylinders, 82, 1030 (1956).

12. B. Molin, Journal of Fluids and Structures, $A$ potential flow model for the drag of shrouded cylinders. 7, 29-38 (1993).

13. J.J. Wang, P.F. Zhang, S.F. Lu, K. Wu, Flow Turbul Combust, Drag reduction of a circular cylinder using an upstream rod. 76, 83-101 (2006).

14. P. A. Baranov, V. L. Zhdanov, S. A. Isaev, V. B. Kharchenko, A. E. Usachov in 2003 published in Fluid Dynamics, Vol. 28, p203.

15. B. Sahin, International Journal of Mechanical Science, Pressure losses in an isolated perforated plate and jets emerging from the perforated plate, 31, 51-61 (1989).

16. A.O. Turhal, B. Cuhadaroglu, Experimental Thermal and Fluid Science, The effects of surface injection through a perforated square cylinder on some aerodynamic parameters, 34, 725-735 (2010).

17. Gozmen, B., Akilli, H., B. Sahin, Passive control of circular cylinder wake in shallow flow. Measurement, 46, 1125-1136 (2013).

18. G. M. Ozkan, V. Oruc, H. Akilli, B. Sahin, Experiments in Fluids, Flow around a cylinder by a permeable outer cylinder in shallow water. 53, 1751-1793 (2012).

19. E. Pinar, G.M. Ozkan, T. Durhasan, M.M. Aksoy, H. Akilli, B. Sahin, Journal of Fluids and Structure, Flow structure around perforated cylinders in shallow water, 55, 52-63 (2015). 